\title{
Análisis de motivaciones hacia prácticas socialmente responsables en pequeñas y medianas empresas turísticas de Ensenada, Baja California
}

Analysis of Motivations towards responsible social practices in small and medium sized enterprises in the tourism sector of Ensenada, Baja California

Análise das motivações para práticas sociais responsáveis em pequenas e médias empresas do setor de turismo de Ensenada, Baja California

Virginia Margarita González Rosales / Universidad Autónoma de Baja California, México / margarita.gonzalez@uabc.edu.mx

Recibido: 8/6/2021 Aceptado: 31/8/2021 Publicado: 29/9/2021

\section{RESUMEN}

En el sector turístico, la responsabilidad social empresarial se encuentra en un momento en el que cada vez más organizaciones la incluyen como parte de las nuevas exigencias sociales que pueden otorgarles un atributo diferencial. Por tal motivo, resulta pertinente conocer las motivaciones que impulsan el desarrollo de dichas prácticas. Mediante la aplicación de encuestas, el análisis de correlaciones de Pearson y una regresión lineal simple, este trabajo tuvo como objetivo analizar la relación entre las motivaciones internas (ética organizacional y competitividad) y externas (presión de y relación con los stakeholders); y las prácticas de responsabilidad social empresarial en el ámbito social, ambiental y económico. A partir de los resultados obtenidos, se identificó que las motivaciones internas presentaron mayor grado de asociación con todas las prácticas de responsabilidad social empresarial ( $r=0.636)$, mientras que entre las externas, la presión de los stakeholders solamente se relacionó con las prácticas medioambientales $(r=0.205)$; y la relación con los stakeholders, con las prácticas económicas $(r=0.238)$.

Palabras clave: correlación, pymes, responsabilidad social empresarial, turismo 


\begin{abstract}
In the tourism sector, corporate social responsibility is at a time when more and more organizations include it as part of the new social demands that can give them a differential attribute. For this reason, it is relevant to know the motivations that drive the development of these practices. Through the application of surveys, Pearson's correlation analysis and a simple linear regression, this work aimed to analyze the relationship between internal motivations (organizational ethics and competitiveness) and external (pressure from and relationship with stakeholders), and corporate social responsibility practices in the social, environmental and economic fields. From the results obtained, it was identified that internal motivations showed a higher degree of association with all corporate social responsibility practices $(r=0.636)$, while among the external ones, stakeholder pressure was only related to environmental practices $(r=0.205)$; and the relationship with stakeholders, with economic practices $(r=0.238)$.
\end{abstract}

Keywords: correlation, corporate social responsibility, SMEs, tourism

\title{
RESUMO
}

No setor de turismo, a responsabilidade social empresarial se encontra em um momento em que cada vez mais as organizações a incorporam às novas demandas sociais que podem Ihes conferir um atributo diferencial. Por isso, é relevante conhecer as motivações que impulsionam o desenvolvimento dessas práticas. Através da aplicação de inquéritos, análise de correlação de Pearson e uma regressão linear simples, este trabalho teve como objetivo analisar a relação entre motivações internas (ética organizacional e competitividade) e externas (pressão e relacionamento com stakeholders); e práticas de responsabilidade social corporativa nas áreas social, ambiental e econômica. Pelos resultados obtidos, identificou-se que as motivações internas apresentaram maior grau de associação com todas as práticas de responsabilidade social corporativa $(r=0.636)$, enquanto entre as externas a pressão dos stakeholders esteve relacionada apenas às práticas ambientais $(r=0.205)$; e o relacionamento com as partes interessadas, com as práticas econômicas $(r=0.238)$.

Palavras chave: correlação, PMEs, responsabilidade social corporativa, turismo

\section{INTRODUCCIÓN}

De acuerdo con Flores et al. (2016), las graves crisis internacionales de la década de los setenta fueron el parteaguas para poner en tela de juicio los planteamientos de desarrollo sobre los que se había fundamentado el crecimiento económico, dando paso a una nueva forma de entendimiento del progreso económico en la que, reconociendo su importancia, se incorporaron las dimensiones ambiental y social, las cuales, junto con la económica, conforman de manera generalizada el concepto de desarrollo sustentable.

En función de ese concepto, y considerando que los cambios en el entorno local depen- 
den de sus mismos actores, las empresas tienen un papel preponderante en el desarrollo sustentable, como consecuencia de su capacidad para impulsar el emprendimiento y de esa manera, potenciar el crecimiento $y$ el desarrollo del territorio donde se ubican (Mora y Martínez, 2018). Es por ello que para contribuir a esta nueva forma de desarrollo surge el concepto de responsabilidad social empresarial (RSE) o responsabilidad social corporativa (RSC), mediante el cual se intenta contribuir a la sostenibilidad de los procesos de desarrollo.

El término responsabilidad social empresarial o corporativa ha evolucionado desde los años cincuenta con Bowen (1953), quien la define como las obligaciones de las empresas para aplicar políticas, tomar decisiones o seguir líneas de acción que sean deseables respecto a los objetivos y valores de la sociedad (Javed et al., 2020). Sin embargo, a partir de 1932, Dodd apunta que los grupos de interés deben ser tenidos en cuenta en el desarrollo de la actividad de las organizaciones, cuestionando ante quiénes estas deben responder y ser responsables, ampliando el grupo de interés o stakeholders hasta la sociedad en general (Vaca, 2012).

El concepto es bastante amplio y hoy en día sigue en constante evolución, visto desde diferentes perspectivas y permeando desde hace muchos años en el ámbito organizacional, al constituir una parte fundamental del desarrollo de muchas empresas (Palacio, 2020). Sin embargo, a pesar de la falta de consenso en una única definición para la academia, su interpretación constituye un factor de vital importancia para ser asumida por las organizaciones, pues de ello dependerá el grado de transversalidad de su aplicación (Licandro et al., 2019). Por lo anterior, en esta investigación se considera a la RSE como la base para que las empresas integren voluntariamente las preocupaciones sociales y ambientales en sus actividades comerciales y en sus relaciones con las partes interesadas (Vergara et al., 2020). De tal manera, a través de este concepto se integran las mismas dimensiones del desarrollo sustentable, considerando también las relaciones con los stakeholders.

En tal sentido Hernández et al. (2017) mencionan que la responsabilidad social en las empresas ha sido denominada, indistintamente, tanto empresarial como corporativa; sin embargo, esta última se relaciona generalmente con las empresas de gran tamaño. De igual modo, Jenkins (2004) concuerda con Habisch y Wegner (2005) en que la connotación «de corporativa» hace referencia a las diferencias culturales que existen entre las grandes, medianas y pequeñas organizaciones, y que dada la dificultad de las pymes para asumir sus responsabilidades sociales, debido al factor tamaño, es preferible el término RSE para ellas. Es por ello que en la investigación se asume la propuesta de estos autores.

El uso del concepto de RSE se ha vuelto cada vez más recurrente, siendo un tema fundamental en la cotidianidad de las empresas de todos los tamaños y sectores (López et al., 
2017). Esto también es mencionado por Ormanza et al. (2020), quienes señalan que la RSE es un modelo aplicable a toda organización, en cualquier actividad o país, para valorar y comparar su aportación hacia el logro de un desarrollo sustentable. En ese mismo orden de ideas, Huerta y Andrade (2021) hacen un estudio en el que analizan la presencia de la RSE dentro de la misión, visión y valores de 100 empresas consideradas como las más importantes de México; encontrando que en 27 diferentes sectores de la actividad empresarial se presentan de alguna manera este concepto o sus dimensiones.

La responsabilidad social es un tema ampliamente abordado desde diferentes posturas y en diversos círculos empresariales. Ello se debe a que las organizaciones se han visto obligadas a incluir, como parte de su gestión, estrategias encaminadas a beneficiar a la sociedad donde se insertan, a partir de las relaciones que tienen con el entorno y sus grupos de interés. Unido a ello deben responder a las políticas tanto locales como mundiales que regulan los impactos sociales, ecológicos y económicos que derivan de su quehacer (Ruiz et al., 2020).

El sector turístico no ha estado exento de dicho abordaje; en efecto, Garay y Font (2013) mencionan que diferentes estudios analizaron el proceso de implementación de la RSC en el sector turístico general, siendo los objetivos de investigación más comunes: el efecto de las medidas introducidas sobre los resultados financieros de las grandes em- presas turísticas y sus relaciones con otras variables, así como las peculiaridades que presentan las pequeñas y medianas empresas de este sector, dejando de lado el estudio de las motivaciones y el grado de implementación de las prácticas de RSC. Las pymes no suelen tener una estructura compleja de dirección y, generalmente, están gestionadas por sus mismos propietarios, los que se orientan hacia la solución del día a día del negocio y cuentan con relaciones informales con sus stakeholders, por lo que difieren notablemente de las grandes empresas del sector. Por consiguiente, las motivaciones y comportamientos responsables pueden diferir de manera considerable entre las grandes empresas y las pymes turísticas.

Teniendo en cuenta lo anterior, se puede decir que es innegable la importancia de las prácticas socialmente responsables en todo tipo de organización, por lo que conocer cuáles son los factores que impulsan a las empresas a llevarlas a cabo resulta pertinente para fomentar su ejercicio, dado que las estrategias corporativas se ven muy afectadas por la cognición y el comportamiento individual de los gerentes (Li et al., 2019). En este sentido, Ruiz et al. (2020), citando a Cristóbal y de la Maza (2012); Blom y Mamia (2006); Alvarado y Currás (2011) y Bhardwaj et al. (2018), consideran que existen dos motivaciones primarias: la primera, una perspectiva ética que permita equilibrar los fines económicos a través de las cuestiones sociales y ambientales sobre las que la empresa impacta; la segunda, relacionada directamente con la rentabilidad, a partir de 
la creación de valor y ventajas competitivas derivadas de la relación empresa-stakeholders.

Por su parte, Li et al. (2019) realizan una profunda revisión teórica de las motivaciones internas y externas, y mencionan que la teoría neoinstitucional proporciona una base práctica y teórica para explorar los mecanismos que impulsan las estrategias de RSE. Sin embargo, presentan patrones contradictorios de influencia: en ocasiones, el incremento de la presión de los stakeholders impulsa las prácticas responsables para obtener beneficios sociales; en otras circunstancias, son los factores internos los que tienen mayor influencia. Por otro lado, sostienen que, desde una perspectiva económica, la RSE motiva una mejor reputación, principalmente cuando las empresas exhiben sus acciones. Sin embargo, esta relación no es consistente, probablemente debido a que las motivaciones económicas no están en consonancia con el carácter voluntario de la RSE. El factor ético también es señalado, encontrando que el liderazgo ético tiene un efecto mediador entre las presiones institucionales y las estrategias organizacionales enfocadas en las prácticas responsables.

De igual forma, Vaca (2012) expone que, aunque las motivaciones que llevan a las organizaciones a la práctica de acciones congruentes con la RSE pueden ser diversas, la mejora de las relaciones con los grupos de interés; la cultura organizacional; la actuación de la competencia o la propia presión de los stakeholders y la búsqueda de un au- mento de la cuota de mercado (Sethi, 1979; Perrow, 1997; Turban y Greening, 1997; Orlitzky et al., 2003; Bertelsmannn Stiftung, 2006; Steger, 2006; Lammertjan, 2006; Rodríguez, 2007) son de relevancia en la organización, ya que el desarrollo de la RSE repercute en ella. Sobre esta base, la autora propone que la conformación de la variable motivaciones se constituye por los constructos variables de competitividad, relación con los stakeholders, presión de los stakeholders y ética-ONG, los mismos que se consideran en este trabajo.

Se considera pertinente el análisis de las motivaciones, tanto internas como externas, para la implementación de prácticas de RSE, ya que los argumentos a favor de esta responsabilidad se relacionan con aquellos que abogan por el desarrollo sustentable, un concepto que sigue siendo válido en la investigación turística. El turismo es una actividad económica con una conexión especial con las comunidades de acogida y sus alrededores, y con el potencial de generar impactos tanto benéficos como perjudiciales para las mismas; además, favorece la concordancia entre el crecimiento económico y las preocupaciones por el medio natural, la cultura y la calidad de vida (Muchazondida, 2016).

Es importante mencionar que, según el Instituto Nacional de Estadística y Geografía (INEGI), en 2018 las actividades relacionadas con el turismo en México reportaron alrededor de 2.3 millones de ocupaciones remuneradas, con un consumo interno de 3222433 
millones de pesos corrientes y un consumo de los turistas residentes fuera del país (consumo emisor) de 272800 millones de pesos, representando el $8.7 \%$ del producto interno bruto total del país (INEGI, 2018). Esta misma situación se presenta en Baja California, donde, de acuerdo a la Secretaría de Turismo del Estado (SECTURE), en 2017 el Estado recibió una afluencia de alrededor de $\mathbf{1 5 . 2}$ millones de visitantes, de ellos 2.7 millones fueron recibidos por el municipio de Ensenada (SECTURE, 2017), donde se realizó este estudio. De manera tal que el crecimiento económico impulsado por el turismo lleva a los Gobiernos a promover políticas públicas hacia el desarrollo local; por esta razón, se debe incluir un abordaje vigente sobre una perspectiva basada en la triple responsabilidad (Pacherres et al., 2020).

Por todo lo antes expuesto, se considera pertinente analizar las motivaciones de los tomadores de decisiones del sector turístico de Ensenada, en particular de las pymes, con el fin de conocer la relación existente entre los factores que impulsan la implementación de las prácticas socialmente responsables y las estrategias desarrolladas en la gestión de las empresas de este sector. Esto se debe a que la RSE pasó de ser meramente un concepto a constituirse en un compromiso y una forma de administración que toda empresa, sin importar su tamaño, debe ser capaz de entender y manejar en su gestión diaria (Herrera et al., 2016, como se citó en Cañizares, 2020). Es por ello que el objetivo de esta investigación consiste en establecer si existe una correlación entre las motivaciones, tanto internas como externas (clasificadas como competitividad, ética, presión de y relación con los stakeholders) y las prácticas de la RSE en los ámbitos económico, social y ecológico, así como descubrir cuáles son las principales motivaciones que estimulan dichas prácticas.

A partir de este primer apartado introductorio, el trabajo continúa su organización con la metodología que describe el proceso seguido, desde la obtención de la información hasta el análisis de los datos, para dar paso al apartado de resultados, donde se busca explicar al lector las relaciones entre las diferentes motivaciones y las prácticas que siguen las empresas. Posteriormente, se discuten los hallazgos y por último, se presentan las conclusiones y recomendaciones finales.

\section{MATERIALES Y MÉTODOS}

Tomando como base al instrumento de Vaca (2012), se realizó una adaptación del mismo hacia el contexto específico de Baja California y se depuró, dejando a las motivaciones como variable independiente, con cuatro dimensiones: competitividad (VC), presión de stakeholders (PS), relación con stakeholders (RS) y ética organizacional (EO). Como variable dependiente, se consideraron las prácticas de responsabilidad social, con tres dimensiones: prácticas ambientales, prácticas sociales y prácticas económicas (figura 1). 
Figura 1. Variables y dimensiones

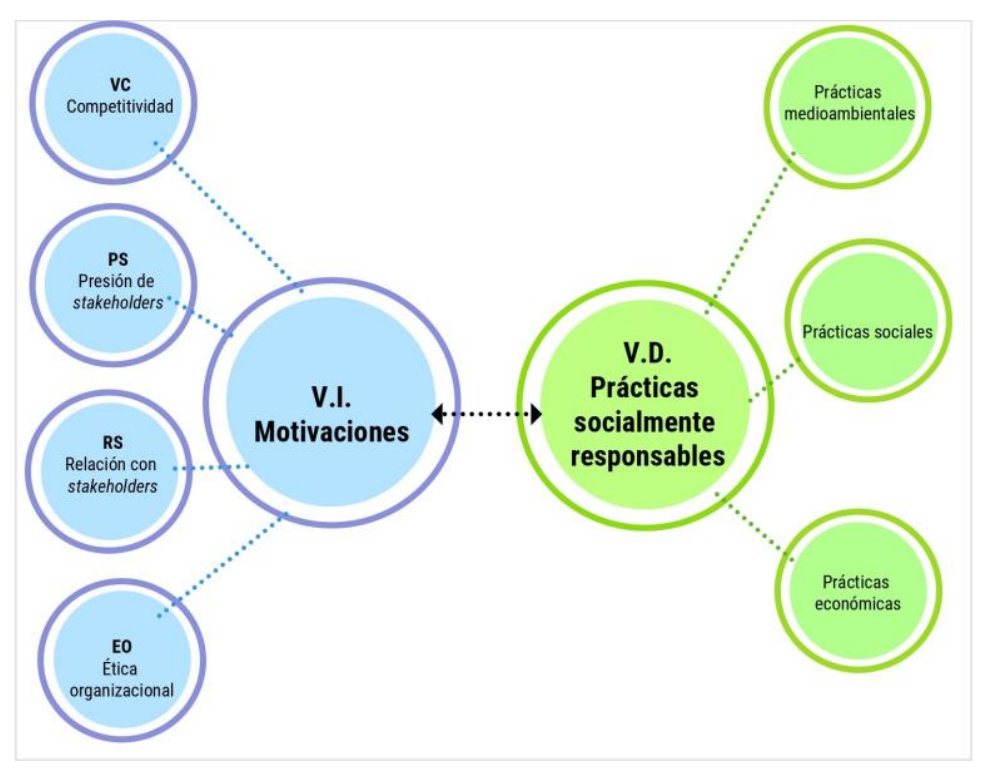

Fuente: Elaboración propia a partir de Vaca (2012).

Una vez adaptado el instrumento de medición, se realizó una prueba piloto en el programa estadístico Statistical Package for the Social Sciences (SPSS) versión 20, posteriormente se hizo un análisis de fiabilidad, obteniéndose un Alfa de Cronbach de 0.894 para el instrumento general, constituido por 40 ítems. Este indicador utiliza para su medición fórmulas que producen coeficientes de fiabilidad que pueden oscilar entre cero (0) y uno (1), donde un coeficiente de cero (0) significa confiabilidad nula y un coeficiente de uno (1) representa un máximo de fiabilidad (Betancourt y Caviedes, 2018). En la presente investigación, la confiabilidad de los factores por Alfa de Cronbach fue considerada satisfactoria, conforme a los parámetros referenciados por la literatura (Hair, 2010 y Pearson, 2013 en Souza et al., 2021). Una vez realizada la prueba piloto, se procedió a aplicar el instrumento a 123 empresas con las que se buscaba representar un universo de 279 que, de acuerdo a la Secretaría de Turismo de Baja California (2014), pertenecen al sector de interés. El criterio de selección se dirigió al personal de nivel medio-alto que labora en empresas del sector turístico localizadas en la ciudad de Ensenada, Baja California. El procedimiento de aplicación fue personal y las proporciones de cuestionarios aplicados por subsector se presentan en la tabla 1. 
Tabla 1. Encuestas aplicadas a los prestadores de servicios turísticos de la ciudad de Ensenada, Baja California, México

\begin{tabular}{lc}
\hline Servicios turísticos & Frecuencia \\
\hline Agencia de viajes & 2 \\
Centro nocturno & 3 \\
Servicio de transporte & 4 \\
Prestador de servicios de naturaleza & 7 \\
Otro & 14 \\
Hotel & 18 \\
Restaurantes y bares & 75 \\
\hline Total & $\mathbf{1 2 3}$ \\
\hline
\end{tabular}

Fuente: Elaboración propia.

El enfoque que sustentó esta investigación es el cuantitativo, en vista de que se realizó un análisis tanto del nivel de asociación como de dependencia entre varias variables mediante correlaciones de Pearson y regresión lineal. En este sentido, de acuerdo con Parisca (2009, como se citó en Díaz, 2017), la correlación de Pearson es un índice estadístico que evalúa la relación lineal entre dos variables, y que, a diferencia de la covarianza, es independiente de la escala de medida. Por su parte, la regresión lineal se refiere a la prueba estadística que describe cómo las variables de respuesta dependen de uno o varios factores. En otras palabras, miden la relación existente entre cierta variable de interés o dependiente y un conjunto de variables explicativas o independientes (Cisneros, 2020).

Con el enfoque previamente mencionado, se procedió a realizar y analizar las siguientes correlaciones de Pearson: motivaciones y prácticas socialmente responsables; motivaciones internas y externas con prácticas de RSC; motivaciones con tipos de prácticas y tipos de motivaciones con tipos de prácticas. Asimismo, se obtuvo la regresión lineal con la siguiente fórmula: prácticas ambientales $=\beta_{0}+\beta_{1}$ motivaciones \pm error. Nuevamente, se utilizó el software SPSS, bajo la premisa de que si el p-valor es menor a lo que se considera estadísticamente significativo (0.05), se rechaza la hipótesis nula $\left(H_{0}\right) y$, por consiguiente, se acepta la hipótesis alternativa $\left(H_{\mathrm{i}}\right)$.

\section{RESULTADOS}

Para poder medir el nivel de asociación entre la variable independiente (motivaciones) y la dependiente (prácticas socialmente responsables) se realizó un análisis de correlación bivariada de Pearson, ya que el indicador estadístico es definido por el coeficiente de correlación $r$ y medido en una escala que oscila entre -1 y +1 . Un resultado de 0 significa que no hay correlación, es decir, el comportamiento de una variable no se relaciona con el comportamiento de la otra. Una correlación perfecta implica un valor de -1 o +1, lo cual indicaría que al conocer el valor de una variable sería posible determinar el valor de la otra. 
Entre más cercano a 1 sea el coeficiente de correlación, mayor la fuerza de asociación (Roy et al., 2019).

Como se presenta en la tabla 2, se encontró una relación positiva y significativa entre las motivaciones y las prácticas de RSE, con una $r=0.424$ ( $p \leq 0.01)$, es decir, una significancia del $99 \%$, lo que indica que existe una correlación significativa de la variable depen- diente en función de la independiente. Para interpretar qué tan fuerte es la correlación, podemos utilizar el criterio de Cohen (1988), quien, para valores absolutos, indica que entre 0.1 y 0.3 representan un efecto pequeño, de 0.3 a 0.5 , un efecto medio, $\mathrm{y} \geq 0.5$, un efecto grande de correlación. En este caso el resultado corresponde a un efecto medio.

Tabla 2. Correlación de Pearson entre las motivaciones y prácticas de responsabilidad social empresarial

\begin{tabular}{ccc}
\hline & Motivaciones & Prácticas de RSE \\
\hline Motivaciones & 1 & $0.424^{* *}$ \\
Prácticas de RSE & $0.424^{* *}$ & 1 \\
\hline Nota: ${ }^{* *}$ La correlación es significativa a un nivel del 0.01 (2 colas).
\end{tabular}

Fuente: Elaboración propia.

Una vez comprobado que existe una correla- encontrándose que la mayor fuerza de asoción entre las motivaciones propuestas y las prácticas de RSE, se quiso averiguar si esta relación se presentaba tanto en las prácticas internas (competitividad y ética organizacional) como en las externas (presión de los ciación se presenta en las internas (tabla 3). Aunque ambas presentan una relación positiva y significativa, las internas reportan una $r=0.636(p \leq 0.001)$, mientras que las exterstakeholders y relación con los stakeholders), nas una $r=0.322(p \leq 0.005)$.

Tabla 3. Correlación de Pearson entre las motivaciones internas y externas y prácticas de responsabilidad social empresarial

\begin{tabular}{lccc}
\hline & Prácticas de RSE & Externas & Internas \\
\hline Prácticas de RSE & 1 & & \\
Externas & $0.207^{*}$ & 1 & \\
Internas & $0.636^{* *}$ & $0.322^{* *}$ & 1 \\
\hline
\end{tabular}

Nota: * La correlación es significativa a un nivel de 0.05 (2 colas); ** La correlación es significativa a un nivel de 0.01 (2 colas).

Fuente: Elaboración propia.

Como siguiente paso en el abordaje de los objetivos, y para verificar la asociación entre las dimensiones de cada variable, nuevamente se llevó a cabo una correlación bivariada de Pearson (tabla 4), en la que se puede observar como la variable motivaciones se relaciona de manera positiva y significativa con cada una de las prácticas 
de RSE, esto es, con las prácticas sociales $(r=0.300, p \leq 0.001)$, con las prácticas ambientales $(r=0.38, p \leq 0.001)$ y con las prácticas económicas ( $r=0.363, p \leq 0.001)$.

En la misma tabla 4, se aprecia que la dimensión competitividad se relaciona positiva y significativamente con las prácticas sociales $(r=0.379, p \leq 0.001)$, ambientales $(r=0.435$, $p \leq 0.001)$ y económicas $(r=0.430, p \leq 0.001)$. Lo mismo sucede con la dimensión ética organizacional, donde encontramos relaciones positivas y significativas con cada uno de los tipos de prácticas de RSE: sociales $(r=0.525, p \leq 0.001)$, ambientales $(r=0.495$, $p \leq 0.001)$ y económicas $(r=0.527, p \leq 0.001)$.

Sin embargo, esto no sucede con las dimensiones presión de los stakeholders y relación con los stakeholders. En el primer caso, encontramos que la presión de los stakeholders solo se relaciona de manera positiva y significativa con las prácticas ambientales $(r=0.205, p \leq 0.005)$, $y$ en el segundo, que la dimensión relación con los stakeholders solo lo hace con las prácticas económicas $(r=0.238, p \leq 0.001)$.

Tabla 4. Correlación de Pearson entre las dimensiones de la variable motivaciones y las dimensiones de la variable prácticas de responsabilidad social empresarial

\begin{tabular}{lcccccccc}
\hline & Motivaciones & Social & Ambiental & Económica & VC & EO & PS & RS \\
\hline Motivaciones & 1 & & & & & & & \\
Social & $0.300^{* *}$ & 1 & & & & & & \\
Ambiental & $0.380^{* *}$ & $0.557^{* *}$ & 1 & & & & & \\
Económica & $0.363^{* *}$ & $0.655^{* *}$ & $0.419^{* *}$ & 1 & & & & \\
VC & $0.636^{* *}$ & $0.379^{* *}$ & $0.435^{* *}$ & $0.430^{* *}$ & 1 & & & \\
EO & $0.536^{* *}$ & $0.525^{* *}$ & $0.495^{* *}$ & $0.527^{* *}$ & $0.552^{* *}$ & 1 & & \\
PS & $0.833^{* *}$ & 0.049 & $0.205^{*}$ & 0.095 & $0.300^{* *}$ & 0.173 & 1 & \\
RS & $0.811^{* *}$ & 0.167 & 0.159 & $0.238^{* *}$ & $0.329^{* *}$ & $0.225^{*}$ & $0.591^{* *}$ & 1 \\
\hline
\end{tabular}

Nota: VC: competitividad; EO: ética organizacional; PS: presión de stakeholders y RS: relación con los stakeholders. * La correlación es significativa a un nivel del 0.05 (2 colas). ** La correlación es significativa a un nivel del 0.01 (2 colas).

Fuente: Elaboración propia.

Para poder conocer el grado de influencia de la variable independiente sobre la dependiente, se realizó un análisis de regresión simple (tabla 5) en el que se puede observar que la fuerza de asociación entre las variables, que se explica por el coeficiente de determinación $\mathrm{R}$, tiene un valor de 0.424 , indicando que el modelo explica el $42.4 \%$ de la varianza. La prueba F, con un 10.263, muestra que las motivaciones influyen positiva y significativamente en las prácticas de RSE, lo cual se confirma a través de la significancia de $0(\beta=0.424, F=10.263, p \leq 0.001)$, con un Durbin Watson con valor de 2.178.

La prueba de Durbin Watson confirma que los errores en la medición de las variables explicativas son independientes entre sí, considerándose independiente si el estadís- 
tico se encuentra entre 1.5 y 2.5 (Camacho, 2006, como se cita en Vilà et al., 2019), por lo que el valor obtenido se aprecia aceptable.

De manera global, puede afirmarse que las motivaciones, medidas a través de las cuatro dimensiones (competitividad, ética organizacional, relación con los stakeholders y presión de los stakeholders), influyen de forma significativa en las prácticas de RSE, entendidas como el conjunto de prácticas económicas, sociales y ambientales en las empresas del sector turístico de Ensenada, Baja California.

Tabla 5. Regresión lineal simple entre la variable independiente (motivaciones) y la dependiente (prácticas de RSE)

\begin{tabular}{|c|c|c|c|c|c|c|c|c|c|}
\hline \multicolumn{10}{|c|}{ Resumen del modelo } \\
\hline \multicolumn{5}{|c|}{ Modelo } & \multicolumn{5}{|c|}{ Estadísticas de cambios } \\
\hline Constante & $\mathrm{R}$ & $\begin{array}{c}\mathrm{R} \\
\text { cuadrado }\end{array}$ & $\begin{array}{c}R \\
\text { cuadrado } \\
\text { ajustado }\end{array}$ & $\begin{array}{c}\text { Error } \\
\text { estándar de } \\
\text { la estimación }\end{array}$ & Cambio en F & gl1 & $\mathrm{g} / 2$ & Sig. Cambio en $F$ & $\begin{array}{l}\text { Durbin- } \\
\text { Watson }\end{array}$ \\
\hline 1 & 0.424 & 0.18 & 0.173 & 10.263 & 26.544 & 1 & 121 & 0.000 & 2.178 \\
\hline \multicolumn{10}{|c|}{ Coeficientes } \\
\hline \multirow{2}{*}{\multicolumn{2}{|c|}{ Modelo }} & \multirow{2}{*}{\multicolumn{3}{|c|}{\begin{tabular}{|c|c}
\multicolumn{2}{|c|}{ Coeficientes no estandarizados } \\
B & Error estándar
\end{tabular}}} & ficientes tipifice & & \multirow[t]{2}{*}{ t } & \multirow{2}{*}{\multicolumn{2}{|c|}{ Sig. }} \\
\hline & & & & & Beta & & & & \\
\hline \multicolumn{2}{|c|}{1} & 62.583 & \multicolumn{2}{|c|}{5.624} & \multirow{2}{*}{\multicolumn{2}{|c|}{0.424}} & \multicolumn{2}{|c|}{11.127} & 0.000 \\
\hline \multicolumn{2}{|c|}{ Motivaciones } & 0.384 & \multicolumn{2}{|c|}{0.074} & & & \multicolumn{2}{|c|}{5.152} & 0.000 \\
\hline
\end{tabular}

Nota: B: coeficiente de regresión parcial estandarizado Beta que expresa la pendiente de la recta de regresión; T: Estadístico T, que permite comprobar si la regresión entre una variable independiente y la dependiente es significativa; Sig: nivel de significancia; gl1: grados de libertad de la primera variable y g/2: grados de libertad de la segunda variable.

Fuente: Elaboración propia.

De lo anterior se deriva que el modelo de la investigación, $Y=\beta_{0}+\beta_{1} \times 1 \pm e$, queda expresado como

$$
\text { Y: } 62.583+0.424 \times 1 \pm 10.263
$$

Donde:

Y: prácticas de RSE

$\beta_{0}$ : constante

$\beta_{1} \times 1$ : motivaciones

e=error
Es posible afirmar que existe influencia de la variable independiente sobre la dependiente con una $\beta=0.424$, como lo muestra la prueba estadística de regresión simple. Lo anterior se comprueba en la figura 2 a través de la representación gráfica de los datos dentro del plano, la que, de acuerdo con Moreno (2008, citado en Vilà et al., 2019), muestra la relación neta entre las variables representadas. 
Figura 2. Diagrama de dispersión de datos de las variables

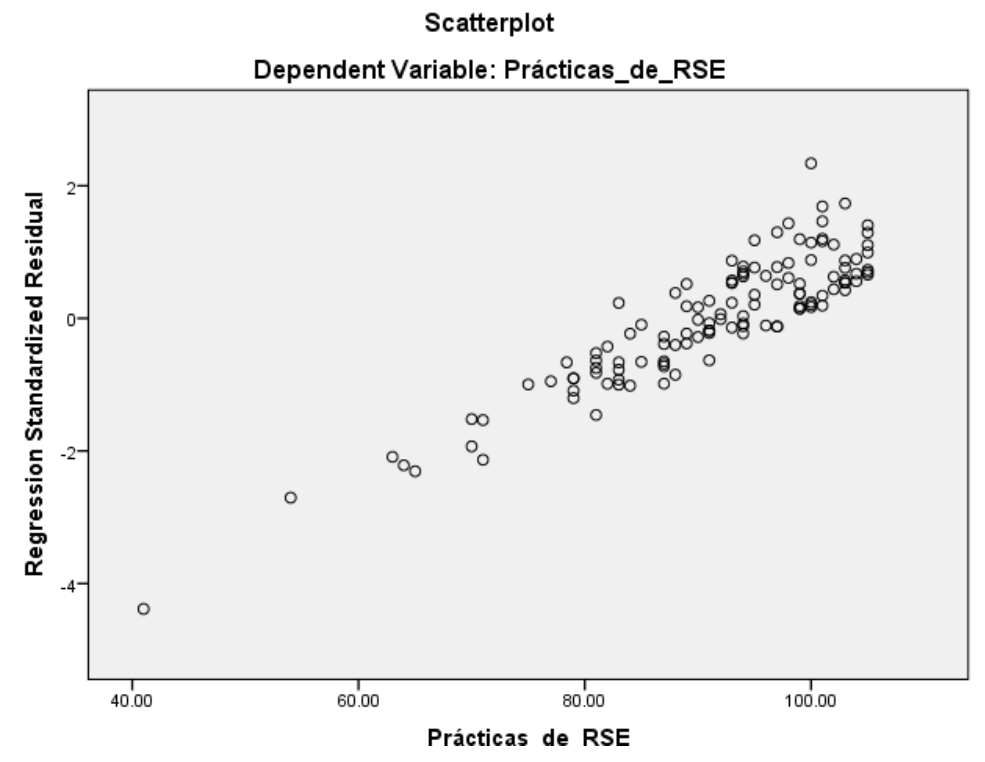

Fuente: Elaboración propia.

\section{DISCUSIÓN}

Los resultados obtenidos ponen de manifiesto que las motivaciones, medidas a través del interés por ser competitivos, de la ética de la organización, de la presión de los stakeholders y de la relación con ellos, se vinculan directamente con las prácticas de RSE en las empresas del sector turístico de Ensenada, y en particular, que dichas motivaciones se ven reflejadas en las acciones, sociales, ambientales y económicas. En esto concuerda Zanga (2020), quien encontró que la variable independiente motivación de los directivos influye positivamente en el nivel de responsabilidad social empresarial, lo cual confirma que las variables y dimensiones seleccionadas como aspectos motivadores fungen como tales en este contexto.

Otro de los hallazgos de esta investigación fue que, dentro de la tipificación de las moti- vaciones, las internas se relacionan con mayor fuerza que las externas. Pese a que ambas fueron estadísticamente significativas, las internas presentan mayor grado de asociación. Esto concuerda con lo que indican Fjørtoft et al. (2020) y Lechuga et al. (2018), para quienes sus resultados sugieren que las motivaciones intrínsecas impulsan más la RSE que las extrínsecas. Estos últimos hacen mención a que las motivaciones internas denotan un compromiso de la empresa, de tal manera que se considera que lo encontrado es consistente con la literatura, y que, además, resulta lógico considerar que la propia ética, cultura organizacional e interés por ser competitivos es un factor determinante no solo para el diseño de estrategias de RSE, sino para la gestión de la empresa en todos sus procesos. Lo visto, también resulta consistente con el hecho de que ambas dimensiones internas se relacionan con las tres tipificaciones de las prácticas de la RSE, 
mientras que las externas solamente se relacionan con las económicas y ambientales.

En ese mismo orden de ideas, dentro de las motivaciones internas, la ética organizacional fue la que presentó cargas más altas y en magnitudes similares al relacionarse con las prácticas de RSE. Esto mismo se presenta en el estudio de Carro-Suárez et al. (2017), quienes reportan que los factores de la cultura organizacional presentan correlaciones significativas con las dimensiones del desarrollo sustentable. Si bien su trabajo se enfoca en la sustentabilidad, las dimensiones y constructos son los mismos. Ello puede deberse a que la implementación de la RSE puede estar más relacionada con razones morales que con la maximización de beneficios. Al respecto, Alvear et al. (2017) encuentran mayor preponderancia en la correlación de la dimensión ética, por lo que consideran que, a través de la evidencia empírica de su investigación, esta dimensión podría interpretarse como la base de todas las demás.

Sin embargo, para este estudio la competitividad resultó también correlacionada con todas las dimensiones de la RSE, y esto puede atribuirse al hecho de que existe una relación ampliamente estudiada entre el desempeño social y el económico (Bernal et al., 2017). Las prácticas de la RSE afectan positivamente la percepción del producto (Robinson y Wood, 2018), además de tener un efecto significativo en la lealtad de los clientes, la marca y el posicionamiento (Akbar et al., 2019). Si bien esto no se ha medido en los consumidores, los tomadores de deci- siones en las empresas que participaron de este estudio pueden considerarlo un objetivo de sus organizaciones $y$, por consiguiente, ser un motivador para la implementación de estas prácticas.

Respecto a las motivaciones externas, la correlación entre la presión de los stakeholders con los aspectos ambientales y los stakeholders con la variable económica evidenció una baja asociación. Ello se debe, en primer término, a la presión que ejercen las organizaciones y la legislación ambiental para el cumplimiento de la normativa correspondiente. En segundo lugar, a que la relación con los stakeholders se vincula exclusivamente con las cuestiones económicas. Resultados similares presentan Haleem et al. (2017), quienes mencionan que sus resultados sugieren que las prácticas de responsabilidad social relacionadas con los proveedores (únicos stakeholders analizados en su trabajo) tienen un efecto de mediación entre la relación de la presión de las partes interesadas y el desempeño ambiental, y entre la presión de las partes interesadas y el desempeño financiero, no encontrándose, efectos de mediación en el desempeño social.

\section{CONCLUSIONES}

A partir de los resultados obtenidos, se puede concluir que existe una correlación entre las motivaciones propuestas y las prácticas de RSE en las pymes del sector turístico; siendo las motivaciones internas las que presentan mayor fuerza de asociación, especialmente, la ética y la cultura organizacional. 
Mientras que las externas tuvieron una baja correlación, presentándose solamente con un tipo de práctica de RSE: la presión de los stakeholders, con las cuestiones ambientales y la relación con los stakeholders con las económicas de manera exclusiva.

Empero, a pesar de que se considera que los resultados son consistentes y concordantes con la literatura, es importante tomar en cuenta diferentes consideraciones. La primera es que el trabajo fue realizado en un entorno específico, Ensenada, Baja California, México, por lo que, considerando su posible transferencia a entornos con características similares, los hallazgos pueden tener una generalización limitada. Además, que el constructo RSE, como se mencionó en los antecedentes, tiene interpretaciones distintas, por lo que las actividades y prácticas que lo constituyen pueden ser conceptualizados de manera diferente en otros contextos. Por último, que las motivaciones pueden variar de acuerdo al sector, tipo de organización (pública o privada), antigüedad, tamaño y demás factores inherentes a su diversidad. Futuras líneas de investigación podrían enfocarse en realizar comparativos entre las motivaciones y dichas diferencias para establecer la aplicabilidad de los resultados en contextos y organizaciones diferentes.

\section{REFERENCIAS BIBLIOGRÁFICAS}

Akbari, M., Mehrali, M., SeyyedAmiri, N., Rezaei, N. y Pourjam, A. (2019). Corporate social responsibility, customer loyalty and brand positioning. Social Responsibility Journal, 16(5), 671-689. https://doi.org/10.1108/SRJ-01-2019-0008

Alvear, L. G., Diaz, R. C., Salas, B. J. y Hernandez, D. R. (2017). Responsabilidad social empresarial en el clúster de muebles en Barranquilla. Revista Espacios, 38(44), 8-25. https://bit.ly/3jEM8LM

Bernal, J., de Nieves, C. y Briones, A. (2017). CSR strategy in technology companies: Its influence on performance, competitiveness and sustainability. Corporate social responsibility and environmental management, 24(2), 96-107. http://doi.org/10.1002/csr.1393

Bertelsmann Stiftung. (Ed.). (2006). Die Gesellschaftliche Verantwortung von Unternehmen. Dokumentation der Ergebnisse einer Unternehmensbefragung der Bertelsmann Stiftung. Bertelsmann Stiftung. https://bit.ly/38CrPlz

Betancourt, A. y Caviedes, I. (2018). Metodología de correlación estadística de un sistema integrado de gestión de la calidad en el sector salud. SIGNOS, 10(2), 119-139. https://doi.org/10.15332/s2145-1389.2018.0002.07

Bowen, H. (1953). Social responsibilities of the businessman. Harper. 
Cañizares, J. (2020). El rol de la responsabilidad social empresarial para las microempresas: ¿qué tanto se cumplen las buenas prácticas? Aibi Revista de investigación, administración e ingeniería, 8(1), 107-115. https://bit.ly/3gZZ5hA

Carro-Suárez, J., Sarmiento-Paredes, S. y Rosano-Ortega, G. (2017). La cultura organizacional y su influencia en la sustentabilidad empresarial. La importancia de la cultura en la sustentabilidad empresarial. Estudios gerenciales, 33(145), 352-36. https://doi.org/10.1016/i.estger.2017.11.006

Cisneros, E. (2020). La natalidad en Ecuador 1990-2017: correlación y regresión con las importaciones de preservativos y anticonceptivos hormonales. Scielo Preprints. https://bit.ly/3CYyWII

Cohen, J. (1988). Statistical power analysis for the behavioral sciences (2nd edition). Academic Press.

de Souza, G., Meireles, E., Mira, V. y Leite, M. (2021). Escala de motivación académica: evidencias de validez y confiabilidad en estudiantes del curso de enfermería. Revista Latino-americana de Enfermagem, 29, e3420. https://bit.ly/3n1JCkQ

Díaz, J. E. (2017). Correlación y regresión lineal de la evaluación tiempo y puntaje con recurso interactivo flash. INNOVA Research Journal, 2(10), 1-8. https://bit.ly/2WNjKPO

Fjørtoft, B., Grimstad, S. y Glavee, R. (2020). Motivations for CSR in the Norwegian maritime cluster: stakeholder perspectives and policy implications. Maritime Policy y Management, 47(8), 1010-1026. https://doi.org/10.1080/03088839.2020.1735654

Flores-Ruiz, D, Bino-Raya, R. E. y de la O, M. (2016). Responsabilidad social en el sector hotelero. Análisis de caso en Córdoba (Argentina). Revista de Globalización, Competitividad y Gobernabilidad, 10(3), 116-135. https://bit.ly/2WNkrrA

Garay, L. y Font, X. (2013). Motivaciones, prácticas y resultados del comportamiento responsable en las pequeñas y medianas empresas turísticas. Revista de Responsabilidad Social de la Empresa, (13), 51-84. https://bit.ly/38DiTm7

Habisch, A. y Wegner, M. (2005). Overcoming the heritage of corporatism. En A. Habisch, J. Jonker, M. Wegner y R. Schmidpeter (Eds.), Corporate social responsibility across Europe ( $1^{\text {a ed.). }}$. Springer (111-125). https://bit.ly/3zJ4uAB

Haleem, F., Farooq, S. y Wæhrens, B. V. (2017). Supplier corporate social responsibility practices and sourcing geography. Journal of cleaner production, 153, 92-103. https://doi.org/10.1016/i. jclepro.2017.03.143 
Hernández, M., Mendoza, J. y Salazar, B. (2017). La responsabilidad social en la empresa familiar. Holos, 5, 174-185. https://bit.ly/3BE1uWN

Huerta-Estévez, A. y Andrade-Estrada, M. G. (2021). Responsabilidad social empresarial en la misión, visión y valores de las principales empresas en México. Estudios Sociales. Revista de Alimentación Contemporánea y Desarrollo Regional, 31(57), 2-22. https://bit.ly/3zMrvml

Instituto Nacional de Estadística y Geografía. (2018). Cuenta satélite del turismo de México (2018). Comunicado de Prensa nro. 700/19. https://bit.ly/2WOukF5

Javed, M., Rashid, M., Hussain, G. y Ali, H. (2020). The effects of corporate social responsibility on corporate reputation and firm financial performance: Moderating role of responsible leadership. Corporate Social Responsibility and Environmental Management, 27(3), 13951409. https://doi.org/10.1002/csr.1892

Jenkins, H. (2004), A critique of conventional CSR theory: An SME perspective. Journal of General Management, 29(4), 37-57. https://doi.org/10.1177/030630700402900403

Lammertjan, D. (2006). Corporate social responsibility in a general equilibrium stock market model: Solving the financial performance puzzle. CCSO Centre for Economic Research, University of Groningen. https://bit.Iy/2YfaTFV

Lechuga, M., Martínez-Martínez, D., Larran, M. y Herrera, J. H. (2018). Understanding the link between socially responsible human resource management and competitive performance in SMEs. Personnel Review, 47(6), 1211-1243. http://doi.org/10.1108/PR-05-2017-0165

Li, X., Gao-Zeller, X., Rizzuto, T. E. y Yang, F. (2019). Institutional pressures on corporate social responsibility strategy in construction corporations: The role of internal motivations. Corporate Social Responsibility and Environmental Management, 26(4), 721-740. https://doi. org/10.1002/csr.1713

Licandro, O., Alvarado-Peña, L. J., Sansores, E. A. y Navarrete, J. E. (2019). Responsabilidad social empresarial: hacia la conformación de una tipología de definiciones. Revista Venezolana de Gerencia, 24(85). https://bit.ly/3n0ikv2

López, J., Ortega, I. y Ortiz, I. (2017). Strategies of corporate social responsibility in Latin America: a content analysis in the extractive industry. AD-Minister, (31), 115-135. https://doi.org/10.17230/ad-minister.31.7

Mora, M. y Martínez, F. (2018). Desarrollo local sostenible, responsabilidad social corporativa y emprendimiento social. Equidad y desarrollo, (31), 27-46. http://dx.doi.org/10.19052/ed.4375 
Muchazondida, J. T. (2016). Beyond reviewing: uncovering the multiple roles of tourism social media users. Journal of Travel Research, 56(3), 287-298. https://doi.org/10.1177/0047287516636236

Orlitsky, M., Schmidt, F. L. y Rynes, S. L. (2003). Corporate social and financial performance: a meta-analysis. Organization Studies, 24(3), 403-441. https://bit.ly/3kRUgrz

Ormaza, J., Ochoa, J., Ramírez, F. y Quevedo, J. (2020). Responsabilidad social empresarial en el Ecuador: abordaje desde la Agenda 2030. Revista de Ciencias Sociales, 26(3), 175-193. https://bit.ly/3gYR7VC

Pacherres, S., Vergara, A. y Sorhegui, R. A. (2020). Responsabilidad social corporativa y el turismo sostenible. Revista científica ECOCIENCIA, 7(2), 1-20. https://doi.org/10.21855/ecociencia. 72.340

Palacio, A. K. (2020). Responsabilidad social empresarial: evolución e importancia dentro de las organizaciones. Revista Colombiana de Contabilidad, 8(15), 75-85. https://bit.ly/2WLmYIA

Perrow, Ch. (1997). Organizing for environmental destruction. Organization and Environment, 10(1), 66-72. https://doi.org/10.1177/0921810697101009

Robinson, S. y Wood, S. (2018). A "good" new brand-What happens when new brands try to stand out through corporate social responsibility. Journal of Business Research, 92, 231241. https://doi.org/10.1016/j.jbusres.2018.07.031

Rodríguez, J. M. (2007). Responsabilidad social corporativa y análisis económico: práctica frente a teoría. Ekonomiaz, (65), 12-49. https://bit.ly/3jFRuX6

Roy-García, I., Rivas-Ruiz, R., Pérez-Rodríguez, M. y Palacios-Cruz, L. (2019). Correlación: no toda correlación implica causalidad. Revista Alergia México, 66(3), 354-360. https://doi.org/10.29262/ram.v66i3.651

Ruiz, L., Camargo, A. y Muñoz, N. (2020). Relación entre responsabilidad social empresarial y rentabilidad: una revisión de literatura. Encuentros, 18(2), 128-141. https://bit.ly/38Fa5wg

Secretaría de Turismo de Baja California. (2014). Agenda de competitividad del destino turístico de Ensenada, B.C. Agendas de competitividad de los destinos turísticos de México. Secretaría de Turismo. https://bit.ly/38H9ZE5

Secretaría de Turismo de Baja California. (2017). Arribo de visitantes. https://bit.ly/3thBP3w

Sethi, S. P. (1979). The role of advocacy advertising in external communications. Journal of General Management, 4(3), 33-44. https://doi.org/10.1177/030630707700400301 
Steger, U. (2006). Building a business case for corporate sustainability. En S. Schaltegger y M. Wagner (Eds.), Managing the business case for sustainability: The integration of social environmental and economic performance. Greenleaf Publishing.

Turban, D. B. y Greening, D. W. (1997). Corporate social performance and organizational attractiveness to prospective employees. Academy of Management Journal, 40(3), 658-672. https://www.jstor.org/stable/257057

Vaca, R. (2012). Responsabilidad social corporativa en las cadenas hoteleras españolas: factores determinantes de su nivel de desarrollo y consecuencias [Tesis de doctorado, Universidad de Huelva]. Repositorio Core. https://core.ac.uk/download/pdf/60655069.pdf

Vergara-Romero, A., Olalla, A., Yturralde, J. M. y Sorhegui, R. (2020). Responsabilidad social corporativa RSC y su impacto en el rendimiento económico de las principales empresas en Ecuador. Revista Espacios, 41(10). https://bit.ly/3tDWvCO

Vilà, R., Torrado-Fonseca, M. y Reguant, M. (2019). Análisis de regresión lineal múltiple con SPSS: un ejemplo práctico. REIRE Revista d'Innovació i Recerca en Educació, 12(2), 1-10. http://doi.org/10.1344/reire2019.12.222704

Zanga, W. (2020). Nivel de desarrollo de la responsabilidad social empresarial y el desempeño empresarial en el sector hotelero del distrito de Tacna, año 2020 [Tesis de grado, Universidad Privada de Tacna]. Repositorio Institucional. https://bit.ly/3tb/YSK 